\title{
PARP-1-dependent RND1 transcription induced by topoisomerase I cleavage complexes confers cellular resistance to camptothecin
}

\author{
Laetitia Mouly ${ }^{1,2}$, Kenza Mamouni ${ }^{1,2}$, Remi Gence ${ }^{1,2}$, Agnese Cristini $^{1,2}$, Julia Cherier ${ }^{1}$, Adrien Castinel ${ }^{2}$, \\ Morgane Legrand ${ }^{2}$, Gilles Favre, ${ }^{1,2}$, Olivier Sordet ${ }^{1}$ and Sylvie Monferran ${ }^{1,2}$
}

\begin{abstract}
RHO GTPases regulate essential functions such as the organization of the actin cytoskeleton. The classic members cycle between an active GTP-bound and an inactive GDP-bound conformation whereas atypical members are predominantly GTP-bound. Besides their well-established role, the classic RHO GTPases RHOB and RAC1, are rapidly induced and/or activated by genotoxic stress and contribute to the DNA damage response. Here we used camptothecin, a selective topoisomerase I (TOP1) inhibitor that stabilizes TOP1 cleavage complexes (TOP1cc), to search for other potential early DNA damage-inducible RHO GTPase genes. We identified that an atypical RHO GTPase, RND1, is rapidly induced by camptothecin. RND1 induction is closely associated with the presence of TOP1cc induced by camptothecin or by DNA lesions that elevate TOP1cc levels such as UV and hydrogen peroxide. We further demonstrated that camptothecin increases RND1 gene transcription and mRNA stability. Camptothecin also increases poly(ADP-ribose) polymerase 1 (PARP-1) activity, whose inhibition reduces RND1 transcription. In addition, overexpression of RND1 increases PARP-1, suggesting a cross-talk between PARP-1 and RND1. Finally, RND1 protects cells against camptothecin-induced apoptosis, and hence favors cellular resistance to camptothecin. Together, these findings highlight RND1 as an atypical RHO GTPase early induced by TOP1cc, and show that the TOP1cc-PARP-1-RND1 pathway protects cells against apoptosis induced by camptothecin.
\end{abstract}

\section{Introduction}

The RHO GTPase family comprises 20 members in human, which can be divided into classic and atypical members ${ }^{1}$. Classic RHO GTPases, such as RHOB and RAC1, cycle between an active GTP-bound and an inactive GDP-bound conformation. Atypical RHO GTPases, such as RND1, are unable to hydrolyze GTP and are

\footnotetext{
Correspondence: Olivier Sordet (olivier.sordet@inserm.fr) or

Sylvie. Monferran (sylvie.monferran@inserm.fr)

${ }^{1}$ Cancer Research Center of Toulouse (CRCT), INSERM, Université de Toulouse,

Université Toulouse III Paul Sabatier, CNRS, Toulouse, France

${ }^{2}$ Faculté des Sciences Pharmaceutiques, Université de Toulouse, Université

Toulouse III Paul Sabatier, Toulouse, France

These authors contributed equally: Olivier Sordet, Sylvie Monferran

Edited by: M. Malewicz
}

therefore in a constitutive active GTP-bound conformation $^{2,3}$. Other atypical members, such as RHOU, and presumably also RHOV, have a high nucleotide exchange rate and hence are assumed to be mainly GTP-bound ${ }^{4}$. Consequently, the tight control of the expression of atypical RHO GTPases is important to precisely tune their activity. GTP-bound RHO GTPases bind to their effectors and regulate pivotal cellular functions, including the organization of the actin and microtubule cytoskeletons, cell adhesion and cell migration ${ }^{5}$.

Besides their canonical roles, the RHO GTPases RAC1 and RHOB have been implicated in the early response to DNA damage. Inhibition or deletion of RAC1 reduces the

\section{(c) The Author(s) 2018}

(c) (i) Open Access This article is licensed under a Creative Commons Attribution 4.0 International License, which permits use, sharing, adaptation, distribution and reproduction in any medium or format, as long as you give appropriate credit to the original author(s) and the source, provide a link to the Creative Commons license, and indicate if changes were made. The images or other third party material in this article are included in the article's Creative Commons license, unless indicated otherwise in a credit line to the material. If material is not included in the article's Creative Commons license and your intended use is not permitted by statutory regulation or exceeds the permitted use, you will need to obtain permission directly from the copyright holder. To view a copy of this license, visit http://creativecommons.org/licenses/by/4.0/. 
DNA damage signaling pathway upon UV light ${ }^{6}$ or ionizing radiation ${ }^{7}$ and, sensitizes cell to ionizing radiation $^{7}$ or to UV-light-induced apoptosis ${ }^{6}$. Unlike RAC1 that is primarily activated in response to DNA damage without change in expression ${ }^{7,8}, \mathrm{RHOB}$ is both induced and activated ${ }^{9-12}$. RHOB induction by genotoxic stress, such as UV light and the topoisomerase I (TOP1) inhibitor camptothecin (CPT), is rapid and relies on increased transcription and/or transcript stability ${ }^{9,10}$. Increased expression of RHOB promotes DNA repair and confers cell resistance to genotoxic stress ${ }^{9}$. At present, it is not known whether, besides RHOB, other RHO GTPases are early DNA damage-inducible genes, at the expression level.

TOP1 solves DNA topological problems that are generated during transcription and replication ${ }^{13}$. It relaxes DNA by forming transient TOP1 cleavage complexes (TOP1cc), which are TOP1-linked DNA single-strand breaks. After DNA relaxation, TOP1cc reverse rapidly, and TOP1 is released as the DNA religates. The transient TOP1cc can be trapped selectively by CPT and its derivatives irinotecan and topotecan, used to treat cancers, which bind at the TOP1-DNA interface ${ }^{14}$. Many DNA alterations including oxidative base damages ${ }^{15,16}$ and UV lesions ${ }^{17,18}$ also interfere with TOP1 nicking-closing reactions and give rise to elevated levels of TOP1cc (see Table 1 in ref. ${ }^{13}$ ). Persistent TOP1cc can lead to the production of DNA double-strand breaks (DSBs) during replication ${ }^{19-21}$ and transcription ${ }^{22-24}$, and ultimately to apoptotic cell death ${ }^{25}$.

An early response to long-lived TOP1cc is the interference with the progression of transcription ${ }^{14,26}$. Indeed, trapping TOP1cc by CPT inhibits transcription elongation with increasing efficiency as the genes become longer and contain more exons ${ }^{27-29}$. However, genes are differentially affected by CPT and a fraction of them, primarily the short and low-expressed genes, are upregulated ${ }^{27,28}$. The mechanisms by which CPT-induced TOP1cc trapping enhances transcription at some genes are largely unknown. Here, we identified RND1 as the first atypical RHO GTPase, which is rapidly induced at the gene level by CPT and DNA damaging agents that indirectly trap TOP1cc, such as hydrogen peroxide $\left(\mathrm{H}_{2} \mathrm{O}_{2}\right)$ and UV light. We found that persistent TOP1cc increase RND1 transcription by a mechanism that depends on poly(ADPribose) polymerase 1 (PARP-1) activity, providing one of the first examples of how stabilized TOP1cc can stimulate gene transcription. Lastly, we found that increased RND1 expression reduces CPT-induced apoptosis, highlighting a protective function for the TOP1cc-PARP-1-RND1 pathway.

\section{Material and Methods}

\section{Drugs, chemical reagents}

CPT, $\mathrm{H}_{2} \mathrm{O}_{2}$, flavopiridol (FLV), actinomycin D, cobalt (II) chloride $\left(\mathrm{CoCl}_{2}\right)$, paclitaxel, methotrexate (MTX), 5aza-2-deoxycytydine (5AZA), trichostatin A (TSA), and the ATR inhibitor VE-821 were obtained from SigmaAldrich, the PARP inhibitor veliparib and the DNA-PK inhibitor NU7441 from Selleckchem, and the ATM inhibitor KU55933 from Millipore. $\mathrm{H}_{2} \mathrm{O}_{2}, \mathrm{CoCl}_{2}$ and actinomycin D were dissolved in water, MTX in $0.1 \mathrm{M}$ sodium hydroxide and the other agents in DMSO.

\section{Cell lines, culture and treatments}

Human osteosarcoma (U2OS), glioblastoma (U87), and colon carcinoma (HCT116) cells, and murine melanoma (B16F10), and embryonic (NIH3T3) cells were obtained from the American Type Culture Collection (ATCC), and cultured in Dulbecco's modified Eagle's medium (DMEM) supplemented with 10\% (v/v) fetal bovine serum. HCT116 cells of each genotype (p53+/+ and $\mathrm{p} 53-/-)$ were kind gifts from Dr. Bert Vogelstein (John Hopkins Kimmel Cancer Center, Baltimore, MD). Primary human lung embryonic WI38 fibroblasts immortalized with hTERT were obtained from Estelle Nicolas (LBCMCP, Toulouse, France) and Carl Mann (CEA, Gif-sur-Yvette, France $)^{30}$ and cultured in modified Eagle's medium (MEM) supplemented with $10 \%(\mathrm{v} / \mathrm{v})$ fetal bovine serum, $1 \mathrm{mM}$ sodium pyruvate, $2 \mathrm{mM}$ glutamine and $0.1 \mathrm{mM}$ non-essential amino acids. In Fig. 2a, cells were irradiated at $500 \mathrm{~J} / \mathrm{m}^{2}$ with a UVB lamp RMX3W system (312 nm) from BioSun (Vilber Lourmat). In all the experiments, mock samples were only treated with the vehicle.

\section{Cell transfection and transduction}

To establish U2OS shRND1 and shCtrl cell lines, 3,000 U2OS cells were transfected using jetPEI reagent (Polyplus), with $1 \mu \mathrm{g}$ of shRNA plasmid with a sequence directed against RND1 mRNA (5'-GGACAGAAAT CCTAGATTATT-3'; QIAGEN) or a control sequence (5'-GGAATCTCATTCGATGCATAC-3'; QIAGEN). 2days after transfection, transfected cells were seeded at low density and treated with puromycin. After 2 to 4 weeks of selection, resistant clonal cells appeared, were removed with cloning cylinder and then amplified.

For transient siRNA transfection in Fig. 2d, U2OS or WI38 hTERT cells were transfected for $48 \mathrm{~h}$ with $50 \mathrm{nM}$ of siRNA duplexes against TOP1 (5'-GGACUCCAUCA GAUACUAUdTdT-3'; QIAGEN) or a non-targeting sequence (SR-CL000-005; Eurogentec) with Dharmafect 4 transfection reagent (GE Healthcare). 
For transient transfection in Fig. 7e, 1.5 million U2OS cells were transfected with $10 \mu \mathrm{g}$ of p-EGFP-RND1 (Addgene) or with $10 \mu \mathrm{g}$ of p-EGFP (Clontech) using jetPEI reagent (Polyplus) according to the manufacturer's protocol. Forty-eight hours after transfection, cells were sorted by FACS in either GFP-positive or GFP-negative RND1.

To establish U2OS RND1-V5 cell lines, 20,000 (for RND1-V5-high) or 40,000 (for RND1-V5-low) U2OS cells were transduced in complete medium with $10 \mu \mathrm{g} / \mathrm{mL}$ of polybrene with lentiviral particles (MOI of 5:1) containing the pLX317-puromycin-RND1-V5 (which contains the CDNA of RND1; Sigma-Aldrich) or a control sequence (tGFP; Sigma-Aldrich). 3 days after transduction, cells were selected with puromycin.

\section{Quantitative reverse transcription-PCR}

Total RNAs were extracted using the RNeasy Plus mini kit (QIAGEN) according to the manufacturer's instructions and the concentration and purity of RNA were determined using the Nanodrop ND-1000. RNAs were reverse transcribed using the iScript cDNA synthesis kit (Bio-Rad). qPCR analyses were performed on a CFX96 real-time system device (Bio-Rad) by using IQ SYBR green Supermix (Bio-Rad) according to the manufacturer's instructions. All samples were analyzed in triplicate, and actin, GAPDH and $28 \mathrm{~S}$ mRNA were used as endogenous controls in the $\triangle \triangle \mathrm{CT}$ analysis. The human $(h)$ and mouse $(m)$ primer pairs used were $h R H O A-\mathrm{FW}$ (5'-TGGAAGATGGCATAACCTGTC-3') and hRHOARV (5'-AACTGGTGGCTCCTCTGG-3'), $h R H O B-F W$ (5'-TTGTGCCTGTCCTAGAAGTG-3') and $h$ RHOB-RV (5'-CAAGTGTGGTCAGAATGCTAC-3'), hRHOC-FW (5'-TGTCATCCTCATGTGCTTCTC-3') and $h R H O C$ -RV (5'-GTGCTCGTCTTGCCTCAG-3'), hRAC1-FW (5'-AGAACACCGAGCACTGAAC-3') and $h R A C 1-\mathrm{RV}$ (5'-ACGCATCTGAGAACTACATAGG-3'), hRAC2-FW (5'-GGACAGCAAGCCAGTGAAC-3') and hRAC2-RV (5'-GGAGAAGCAGATGAGGAAGAC-3'), hRAC3-FW (5'-GTGATGGTGGACGGGAAAC-3') and hRAC3-RV (5'-CACTTGGCACGAACATTCTC-3'), hRHOG-FW (5'-CCGCTCTCACTTCCTTCTC-3') and hRHOG-RV (5'-ACCACCACGCACTTGATG-3'), hCDC42-FW (5' -GTCAAGTATGTGGAGTGTTCTG-3') and $h C D C 42$ -RV (5'-CACCTGCGGCTCTTCTTC-3'), hRHOJ (QIA GEN; QT00092078), hRHOQ-FW (5'-TATGCCAAC GACGCCTTC-3') and hRHOQ-RV (5'-GCCGTGT CA TAGAGTCCTAG-3'), hRHOD-FW (5'-GATTG GAGCCTGTGACCTAC-3') and hRHOD-RV $\left(5^{\prime}\right.$ -GTAATCCGCCGCCAGAAG-3'), hRHOF-FW (5'-CA GACAGACCTCACGACAG-3') and hRHOF-RV (5' -AGTTCCAGAATGTTCCAAGAG-3'), hRHOU-FW (5' -CGGTGGTGTCTGTGGATG-3') and hRHOU-RV (5' -GAAGATGTCTGTGTTGGTGTAG-3'), hRHOV-FW
(5'-CATAGCAAGTAGTAGGCAGGAG-3') and $h R H O V$ -RV (5'-TCAGAGTGGGCAGTTAGAGG-3'), hRND1aFW (5'-GCAAGTGTTAGCGAAGGA-3') and hRND1aRV (5'-GCAGAGTGGACGGACA-3'), hRND1b-FW (5' -CGCTCTGAACTCATCTCTTC-3') and $h R N D 1 b-\mathrm{RV}$ (5'-CCATTCCTGTCTCCTTCCAA-3'), mRND1-FW (5'-CAGTTGGGCGCAGAAATCTAC-3') and mRND1 -RV (5'-TGGGCTAGACTTGTTCAGACA-3'), hRND2 (QIAGEN QT00219891), hRND3-FW (5'-CCTGCTCC TCTCGCTCTC-3') and hRND3-RV (5'-TCTGGCTGG CTCTTCTCTC-3'), hRHOH-FW (5'-TTCACCTCCGA GACCTTCC- $\left.3^{\prime}\right)$ and $h R H O H-R V$ (5'-GCCACA GAGTAGCACATCAG-3'), hRHOBTB1-FW (5'-TGG AGCGTTCTCGGGATGT-3') and hRHOBTB1-RV (5'CGAAAAACAGAGGACCACAACA-3'), hRHOBTB2FW (5'-CAGCCAGCTTTGACGTGTG-3') and $h R H$ OBTB2-RV (5'-TTGCCCCGTAAGATCCCGT-3'), actin -FW (5' -TCCCTGGAGAAGAGCTACGA-3') and actinRV (5'-AGGAAGGAAGGCTGGAAGAG-3'), GAPDHFW ( 5'-TGCACCACCAACTGCTTAGC-3') and GAPD $H$-RV ( 5'-GGCATGGACTGTGGTCATGAG-3') and 28S-FW (5'-TCGCTGGGTCTTGGATGT-3') and 28SRV (5'-AGCAGATTGTGACAGACCATTCC-3'). $h$ RND $1 a$ primers were designed at exons 1 and 3 and $h$ RND $1 b$ primers at exon 5. $h$ RND1 $a$ primers were used to perform experiments showed in Fig. 1a, c-e (for U87 and HCT116 cells), Figs. 1g, $2 \mathrm{a}$ (for UVB) and Fig. $2 \mathrm{~b}$. $h$ RND1 $b$ primers were used to perform all the other RT-qPCR experiments.

\section{Cell extracts and immunoblotting}

Whole cell extracts were obtained by lysing cells in $1 \%$ SDS and $10 \mathrm{mM}$ Tris- $\mathrm{HCl}(\mathrm{pH} 7.4)$ supplemented with protease inhibitors (Sigma-Aldrich) and phosphatase inhibitors (Halt phosphatase inhibitor cocktail; ThermoFisher). Viscosity of the samples was reduced by brief sonication. To detect PAR, cell extracts were performed as described previously ${ }^{31}$. To detect ATM, ATM-pS1981, DNA-PK and DNA-PK-pS2056, cell extracts were prepared as previously described ${ }^{22}$. Proteins were separated by SDS-PAGE and immunoblotted with the following antibodies: anti-actin (MAB1501; Millipore), anti-ATM (ab32420; Abcam), anti-ATM-pS1981 (ab81292; Abcam), anti-cleaved caspase-3 (\#9664; Cell Signaling), anticaspase-9 (\#9502; Cell Signaling), anti-Chk1 (sc84081; SantaCruz), anti-Chk1-pS345 (\#2348; Cell Signaling), anti-DNA-PK (ab1832; Abcam), anti-DNA-PK-pS2056

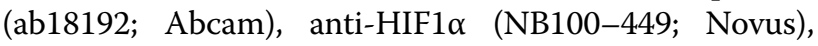
anti-PAR (AM80-100UG; Millipore), anti-p53 (\#48818; Cell Signaling), anti-p53-pS15 (\#9284, Cell Signaling), anti-PARP-1 (\#9542; Cell Signaling), anti-TOP1 (ab109374; Abcam), anti-Tubulin (T5168; SigmaAldrich), anti-V5 tag (46-0705; Invitrogen). Immunoblotting was revealed by chemiluminescence using 


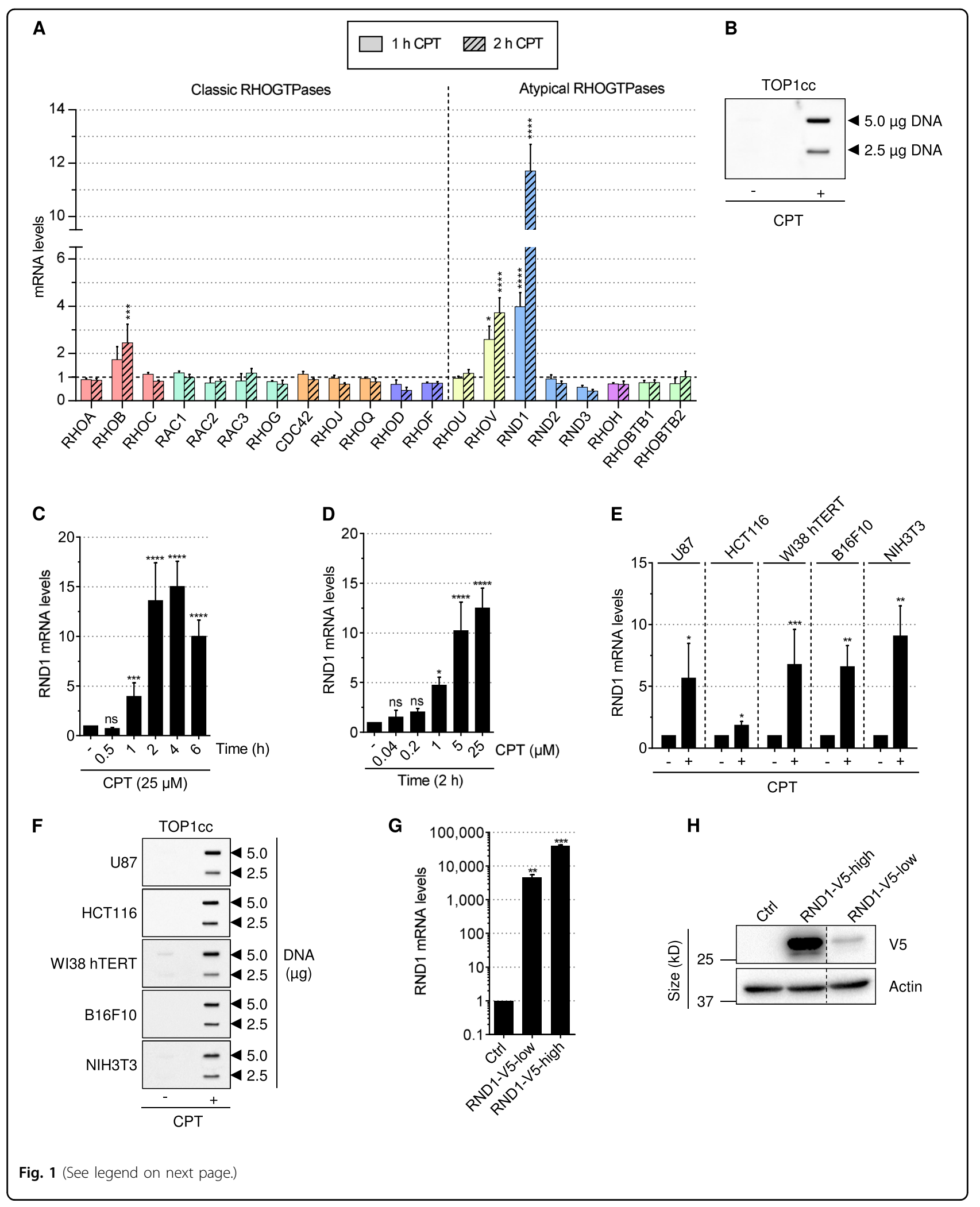


(see figure on previous page)

Fig. 1 Rapid induction of RND1 transcripts by CPT. a RT-qPCR analysis of RHO GTPase mRNA in U2OS cells treated with $25 \mu \mathrm{M}$ CPT for the indicated times. Data were normalized to that of untreated cells, which was denoted by the dashed line (means \pm SEM, $n \geq 3$ ). ${ }^{*} P<0.05$, ***P $P<0.001$, ${ }^{* * * *} P<0.0001$ by two-way ANOVA. Colors are RHO GTPase sub-families. b Detection of TOP1cc in U2OS cells treated with $25 \mu \mathrm{M}$ CPT for $1 \mathrm{~h}$. Two concentrations of genomic DNA (5 and $2.5 \mu \mathrm{g}$ ) were probed with an anti-TOP1cc antibody. $\mathbf{c}, \mathbf{d}$ RT-qPCR analysis of RND1 mRNA in U2OS cells treated for the indicated times with $25 \mu \mathrm{M}$ CPT (c), and with the indicated CPT concentrations for $2 \mathrm{~h}(\mathbf{d})$. The data are expressed as means \pm SD for $n \geq 3,{ }^{*} P$ $<0.05,{ }^{* *} P<0.001,{ }^{* * *} P<0.0001$ by one-way ANOVA. e, f Cells were treated with $25 \mu \mathrm{M}$ CPT for $2 \mathrm{~h}$ (U87, HCT116, and WI38 hTERT cells) or $4 \mathrm{~h}$ (B16F10 and NIH3T3 cells). (e) RT-qPCR analysis of RND1 mRNA (means \pm SD, $n \geq 3$ ). ${ }^{*} P<0.05,{ }^{* *} P<0.01,{ }^{* * *} P<0.001$ by unpaired $t$ test. f Detection of TOP1cc. Two concentrations of genomic DNA (5 and $2.5 \mu \mathrm{g}$ ) were probed with an anti-TOP1cc antibody. $\mathbf{g}, \mathbf{h} \cup 2 \mathrm{OS}$ were stably expressing V5-tagged RND1 at low (RND1-V5-low) or high levels (RND1-V5-high) or EGFP (ctrl). g RT-qPCR analysis of RND1 mRNA (means \pm SD, $n=3$ ). ${ }^{* *} P<0.01,{ }^{* * *} P<$ 0.001 by unpaired $t$ test. $\mathbf{h}$ Western blotting analysis of $\mathrm{V} 5$ tag. Actin: loading control. Dashed lines indicate that intervening wells have been spliced out. Ns, not significant.

ChemiDoc MP System (Bio-Rad). Quantification of protein levels was done with Image Lab software (version 4.1).

\section{WST-1 cell viability assays}

GFP-positive and GFP-negative RND1 sorted cells (Astrios, Beckman) were immediately seeded in triplicate into 96-well microplates at a density of 1,000 cells per well. Twenty-four hours after plating, cells were treated with increasing concentrations of CPT (from $1.6 \mathrm{nM}$ to $25 \mu \mathrm{M}$ ) and cultured for $72 \mathrm{~h}$. The WST-1 reagent (Roche Diagnostics) was then applied for $1 \mathrm{~h}$ at $37^{\circ} \mathrm{C}$. The formazan dye was quantified at $450 \mathrm{~nm}$ using a plate reader (FLUOstar Optima, BMG Labtech). Data were expressed as the percentage of cell survival (mean \pm SD of treated cells normalized to the mean \pm SD of untreated cells, which was set to $100 \%$ ).

\section{Clonogenic assays}

Three hundred U2OS shCtrl or shRND1 cells were treated with increasing concentrations of CPT (from 1.25 $\mathrm{nM}$ to $20 \mathrm{nM}$ ). Ten days after CPT treatment, cells were fixed with 3.7\% paraformaldehyde (Sigma-Aldrich) and stained with $1 \%$ crystal violet (Sigma). Colonies containing more than 50 cells were counted.

\section{Nascent RNA transcripts analysis}

Nascent RNAs were labeled and captured using the Click-iT Nascent RNA capture kit (Life Technologies) according to the manufacturer's instructions. A 2-h 5ethynyl uridine (EU) pulse at the concentration of $0.2 \mathrm{mM}$ was performed to label nascent RNAs. Three to seven $\mu \mathrm{g}$ of total RNA was used for the Click reaction.

\section{Luciferase reporter assay}

To study the activity of $R N D 1$ promoter, three plasmids were used: pGL3-basal promoterenhancer1RND1-lucF (kindly provided by Dr Tan, Tianjin, China), pGL3basalpromoterRND1-lucF and pGL3promoterenhancer2RND1-lucF (kindly provided by $\mathrm{Dr}$ Minami, Tokyo, Japan). Using jetPRIME reagent
(Polyplus), 60,000 U2OS cells were transiently cotransfected with $2 \mu \mathrm{g}$ pGL3-RND1 promoter plasmid and $20 \mathrm{ng}$ of pRL-CMV (Promega). Luciferase activities were measured $24 \mathrm{~h}$ after transfection by using the Dual Luciferase assay system (Promega). All data were normalized by Renilla luciferase luminescence derived from the cotransfected pRL-CMV as described previously ${ }^{32}$.

\section{Meta-analysis of RND1 mRNA expression}

This analysis was performed using the online NextbioResearch tools (http://www.nextbio.com/). We collected RND1 mRNA expression fold-change after treatment with $\mathrm{CPT}$ or derivatives in different cancer cells (OCI-LY3 cells: diffuse large B cell lymphoma; MCF-7: breast cancer cells; PC3: prostate cancer cells; HCT116: colon cancer cells) or tissue (bone marrow from rats) from five gene expression datasets. GEO accession numbers of gene expression datasets in order of appearance: GSE63902 ${ }^{33}$; GSE51068 ${ }^{34}$; GSE18552 $2^{35}$; GSE5258 ${ }^{36}$ and GSE37352 ${ }^{27}$.

\section{Detection of TOP1 cleavage complexes}

Cellular TOP1 cleavage complexes (TOP1cc) were detected as previously ${ }^{22}$, except that immunoblotting was revealed with a mouse anti-TOP1cc from Millipore $(\mathrm{MABE} 1084)^{37}$ in Figs. 1b, f, 4e and Supplementary Figure 2a.

\section{Flow cytometry}

For sub-G1 analysis, cells were fixed with 70\% ethanol, incubated with RNase A (Sigma-Aldrich) and stained with propidium iodide (PI; Molecular probes). The stained cells were analyzed on a BD Accuri C6 flow cytometer (BD Biosciences). Analysis was performed with the BD Accuri C6 flow cytometer software.

\section{Immunofluorescence}

Fifteen thousand U2OS cells were seeded on glass coverslips. After treatment, cells were washed with PBS, fixed in $3.7 \%$ paraformaldehyde for $10 \mathrm{~min}$, and permeabilized with $0.2 \%$ Triton $\mathrm{X}-100 / 1 \%$ bovine serum albumin 

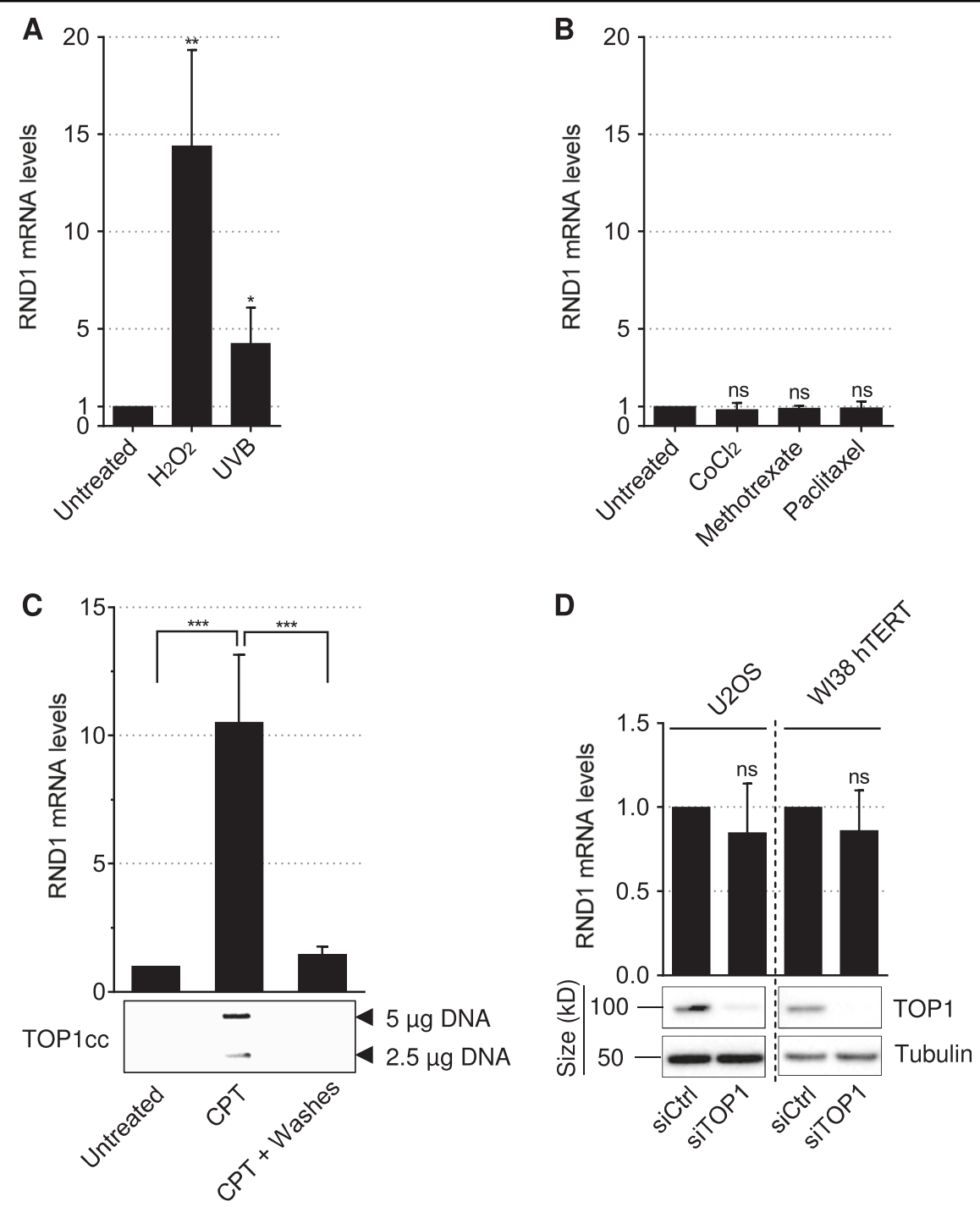

Fig. 2 RND1 transcripts are closely associated with the presence of TOP1cc. $\mathbf{a}, \mathbf{b}$ RT-qPCR analysis of RND1 mRNA in U2OS cells treated for $2 \mathrm{~h}$ with $1 \mathrm{mM} \mathrm{H}_{2} \mathrm{O}_{2}$ or irradiated with $500 \mathrm{~J} / \mathrm{m}^{2} \mathrm{UVB}(\mathbf{a})$, or treated with $100 \mu \mathrm{M} \mathrm{CoCl}_{2}, 50 \mu \mathrm{M}$ methotrexate or $10 \mu \mathrm{M}$ paclitaxel (b). Treatment with CPT has been performed in parallel as a positive control (not shown). Data are expressed as means \pm SD for $n=3,{ }^{*} P<0.05$, ${ }^{* *} P<0.01$ by unpaired $t$ test. c U2OS cells were treated with $25 \mu \mathrm{M}$ CPT for $2 \mathrm{~h}$ and washed and cultured in CPT-free medium (CPT + Washes) for $2 \mathrm{~h}$ to allow reversion of TOP1cc. Top panel: RND1 mRNA was analyzed by RT-qPCR (means $\pm \mathrm{SD}, n=3$ ). ${ }^{* *} P<0.001$ by one-way ANOVA. Bottom panel: Detection of TOP1cc. Two concentrations of genomic DNA ( 5 and $2.5 \mu \mathrm{g}$ ) were probed with an anti-TOP1 antibody. $\mathbf{d}$ U2OS and WI38 hTERT cells were transfected with siRNAs against TOP1 (siTOP1) or against a control sequence (siCtrl). Bottom panel: efficiency of the siRNA determined by Western blot. Tubulin: loading control. Top panel: RND1 mRNA was analyzed by RT-qPCR (means $\pm S D, n=3$ ). Ns, not significant.

(BSA)/PBS buffer for 5 min. Cells were incubated with $10 \%$ BSA for 30 min to block non-specific binding before incubation with anti-tubulin primary antibody (clone B-51-2; Sigma) diluted in 5\% BSA/PBS buffer for $2 \mathrm{~h}$. After washes, cells were incubated with secondary antibody (Alexa Fluor 594 Phalloidin; Thermo Fisher Scientific) diluted in 5\% BSA/PBS buffer for $1 \mathrm{~h}$. After washes, slides were mounted using a mowiol mounting solution containing 4',6'-diamino-2-phenylindole counterstain the DNA. Slides were visualized at room temperature by using an inverted confocal microscope (LSM 780; Carl Zeiss).

\section{Results}

RND1 transcripts are rapidly induced by CPT

To determine the RHO GTPases that are induced early in response to $\mathrm{CPT}$, we treated human osteosarcoma U2OS cells for short times ( $1 \mathrm{~h}$ and $2 \mathrm{~h}$ ), and analyzed 
RHO GTPase mRNA expression by reverse transcription followed by qPCR (RT-qPCR) (Fig. 1a). CPT efficiently induced TOP1cc in U2OS cells (Fig. 1b) as previously reported $^{38}$. Among the RHO GTPase family, the two atypical members $R N D 1$ and $R H O V$, were increased by CPT with RND1 displaying an approximately 4 and 12 folds' increase after $1 \mathrm{~h}$ and $2 \mathrm{~h}$, respectively, and $R H O V$ an approximately 3 and 4 folds' increase (Fig. 1a). $R H O B$ also increased under these conditions (Fig. 1a), as previously reported ${ }^{9}$. Among the two newly identified RHO GTPases, RND1 and RHOV, which are induced early by CPT (Fig. 1a), we further characterized RND1.

Kinetics of RND1 mRNA induction in U2OS cells showed that at $25 \mu \mathrm{M}$ of CPT, RND1 increased within $1 \mathrm{~h}$ and reached a plateau after $2 \mathrm{~h}$ (Fig. 1c). To investigate whether the induction of RND1 mRNA was dose-dependent, cells were treated for $2 \mathrm{~h}$ with increasing CPT concentrations. RND1 induction was detected at $1 \mu \mathrm{M}$ and increased with increasing concentrations of CPT (Fig. 1d). RND1 induction was also observed in other human cell lines (glioblastoma U87, colon carcinoma HCT116, primary lung WI38 hTERT), and mouse cell lines (melanoma B16F10, embryonic NIH3T3) treated with CPT (Fig. 1e). Under these conditions, CPT efficiently induced TOP1cc in all these cell lines (Fig. 1f). Meta-analysis of microarray databases further supports the increase of RND1 mRNA levels after short treatment with CPT or its water-soluble derivatives, topotecan and irinotecan, in human and rodent cell lines, and in tissues (Supplementary Figure 1A). In contrast, the two RND1 homologs, RND2 and RND3 were not induced after a short treatment with $\mathrm{CPT}$ in the cell lines analyzed (Fig. 1a, Supplementary Figure 1B, C).

As other groups ${ }^{39}$, we could not find or generate highaffinity antibodies that react specifically with endogenous RND1. Therefore, to determine whether the increase in RND1 transcript levels could be associated with an increase in RND1 protein levels in CPT-treated cells, we generated U2OS cells stably expressing low or high levels of V5-tagged RND1 transcripts (Fig. 1g). Cells with low and high levels of RND1 transcripts (Fig. 1g) expressed low and high levels of RND1-V5 protein (Fig. 1h), respectively, suggesting that increasing $R N D 1$ transcript expression also increases RND1 protein levels. Altogether, these results identify RND1 as a new early-inducible RHO GTPase gene in response to CPT.

\section{RND1 transcripts are closely associated with the presence of TOP1cc}

CPT has for sole cellular target the TOP1 $1 \mathrm{cc}^{14}$. To assess whether TOP1cc stabilization by CPT primes the increase of RND1 mRNA levels, we examined whether other agents that induce TOP1cc would also induce RND1. Oxidative- and UV-mediated DNA lesions give rise to elevated levels of TOP1cc (see Table 1 in ref. ${ }^{13}$ ). As a result, $\mathrm{H}_{2} \mathrm{O}_{2}$ and UV light induce cellular TOP1cc (Supplementary Figure $2 \mathrm{~A})^{18,40}$. Figure 2a shows that both agents increased RND1 mRNA levels. Conversely, agents that do not induce TOP1cc, including the hypoxiamimicking agent cobalt (II) chloride $\left(\mathrm{CoCl}_{2}\right)$, the dihydrofolate reductase inhibitor methotrexate, and the tubulin inhibitor paclitaxel, did not increase RND1 (Fig. 2b), under conditions where they exert their expected biological effects (for $\mathrm{CoCl}_{2}$, see Supplementary Figure 2b; for methotrexate, see Supplementary Figure 2c; for paclitaxel, see Supplementary Figure 2d).

Because CPT-induced TOP1cc are reversible ${ }^{41}$, we further examined RND1 transcripts following CPT removal. After termination of the CPT treatment, RND1 mRNA returned to their baseline levels (Fig. 2c, top panel) as TOP1cc reversed (Fig. 2c, bottom panel). Because the stabilization of TOP1cc decreases TOP1 activity leading to topological stress ${ }^{14}$, we have examined RND1 induction in TOP1-depleted cells. Figure $2 \mathrm{~d}$ shows that siRNAmediated depletion of TOP1 in U2OS and WI38 hTERT cells did not increase RND1 mRNA levels, suggesting that TOP1cc rather than inhibition of TOP1 activity promote $R N D 1$ induction. Collectively, these results indicate that the increase of $R N D 1$ transcripts is closely associated with the presence of TOP1cc.

\section{CPT increases RND1 transcription and RND1 transcript stability}

The early increase of RND1 mRNA in CPT-treated cells could depend on an increase in transcription and/or in transcript stability. Analysis of RND1 transcription by capture of nascent transcripts followed by RT-qPCR showed that CPT increased by approximately 20 folds the transcription of RND1 gene (Fig. 3a). This increase fully reversed after the removal of CPT (Fig. 3a), indicating that $R N D 1$ transcription is closely related to the presence of TOP1cc. To determine whether the increase of RND1 transcription in CPT-treated cells would depend on the activity of its promoter, we measured the activity of a luciferase reporter gene placed under the control of the RND1 minimal promoter either alone or together with a proximal or a distal enhancer region ${ }^{42,43}$. Fig. 3b shows that CPT did not increase luciferase activity in cells transfected with each of these constructs, suggesting that the increase in RND1 transcription by CPT might not primarily depend on an increased activity of its minimal promoter and the tested enhancers.

Next, we compared the stability of RND1 mRNA between untreated and CPT-treated cells. Experiments performed in the presence of the transcription inhibitor flavopiridol showed that the half-life of RND1 mRNA was greatly prolonged in CPT-treated cells (Fig. 3c). Similar results were obtained with the transcription inhibitor 


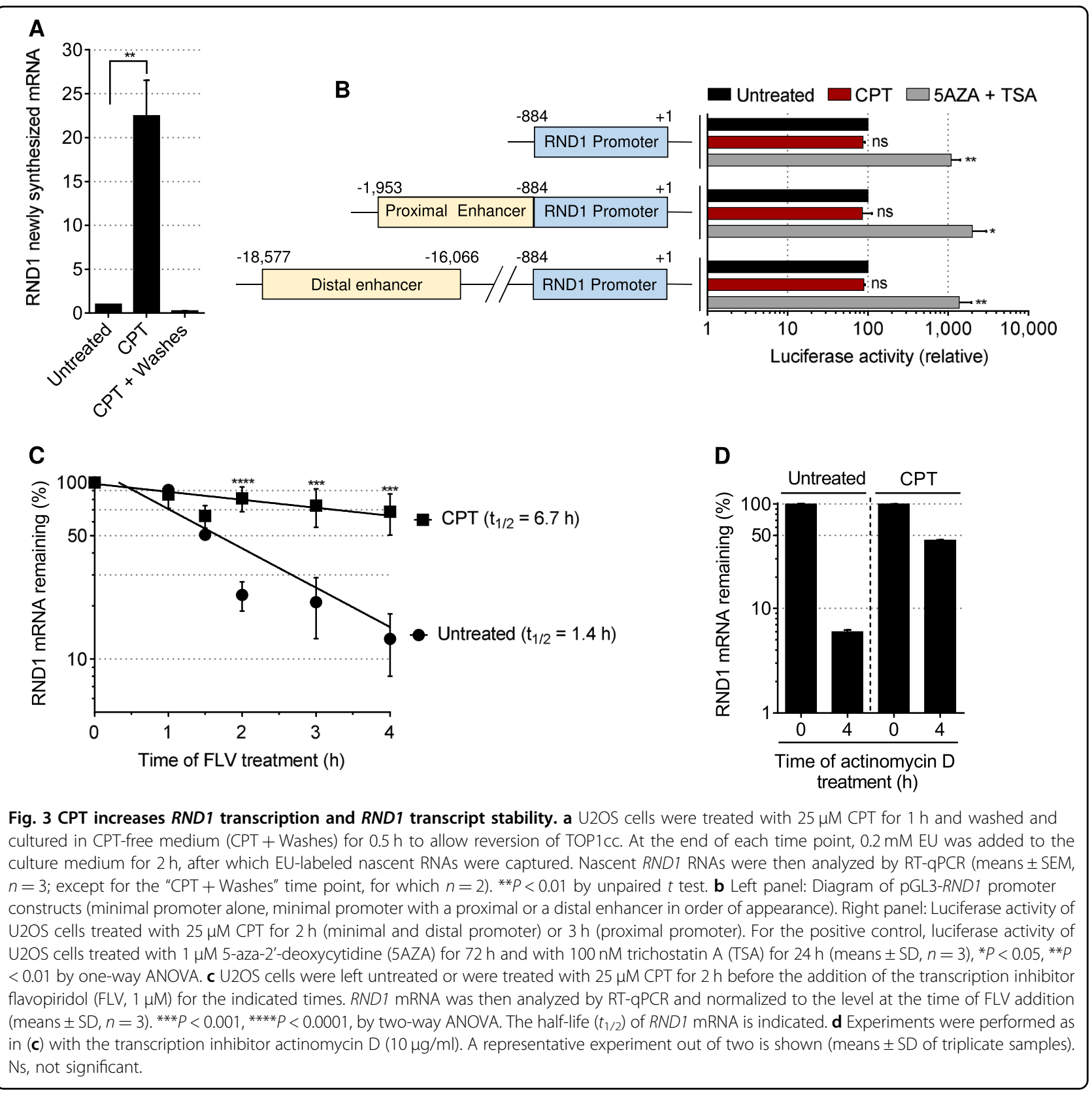

actinomycin D (Fig. 3d). Upon exposure to CPT, TOP1 has been reported to be degraded over time in a transcription-dependent manner ${ }^{22,44,45}$. Hence, the use of transcription inhibitors to analyze the lifespan of RND1 mRNA in CPT-treated cells is likely to sustain the levels of TOP1cc during the time course of these experiments, which might contribute to further increase the half-life of RND1 mRNA. Altogether, these data indicate that the increase of $R N D 1$ transcript levels in response to CPT is associated with an increase in both RND1 transcription and RND1 transcript stability.

\section{PARP-1 increases RND1 transcription in response to TOP1Cc}

PARP-1 can promote gene transcription ${ }^{46-49}$ and transcript stability $^{50}$ via the addition of poly(ADP-ribose) residues (PAR) on proteins, an activity named PARylation. Because a short time CPT treatment increases PARP-1 

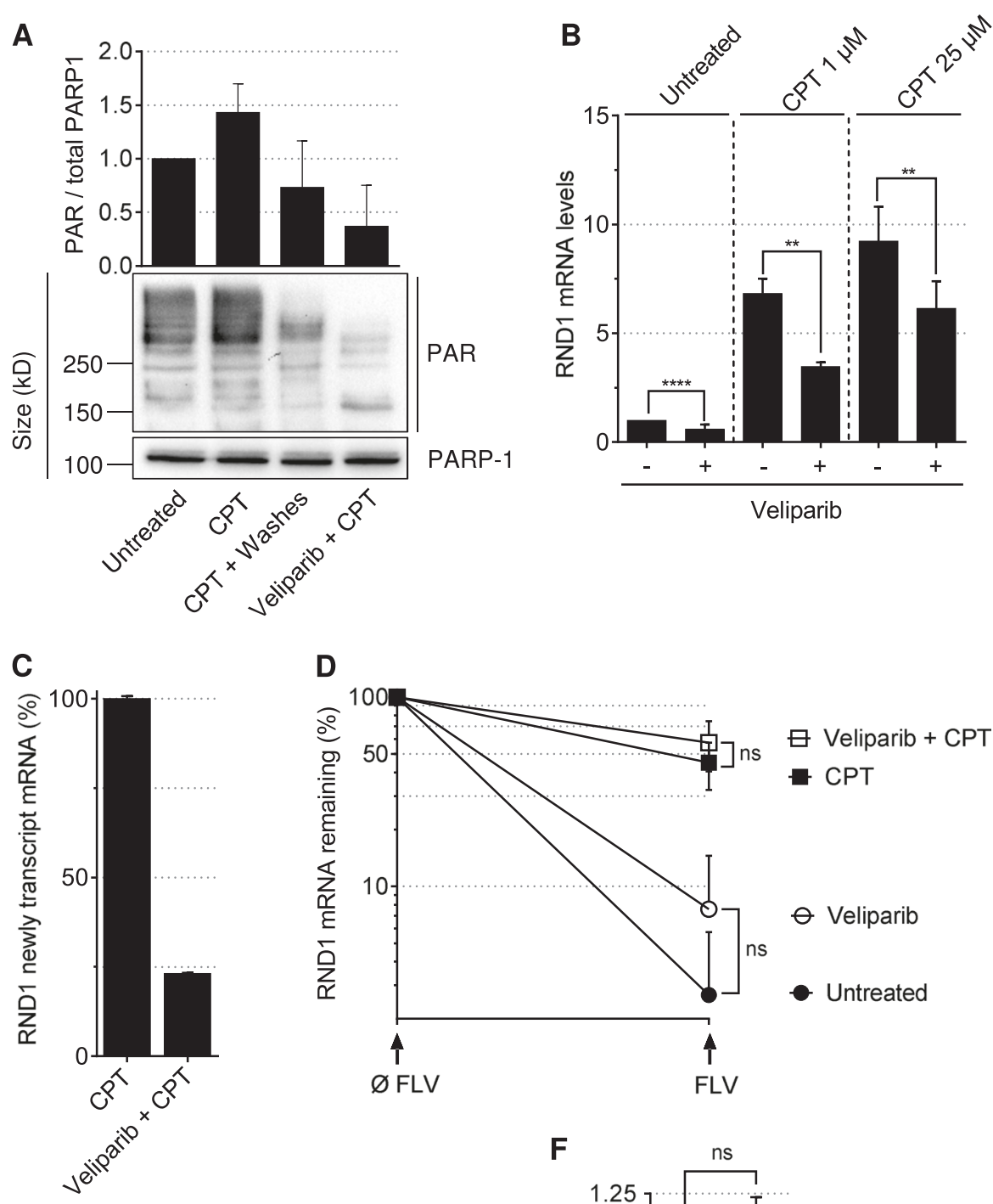

\section{D}
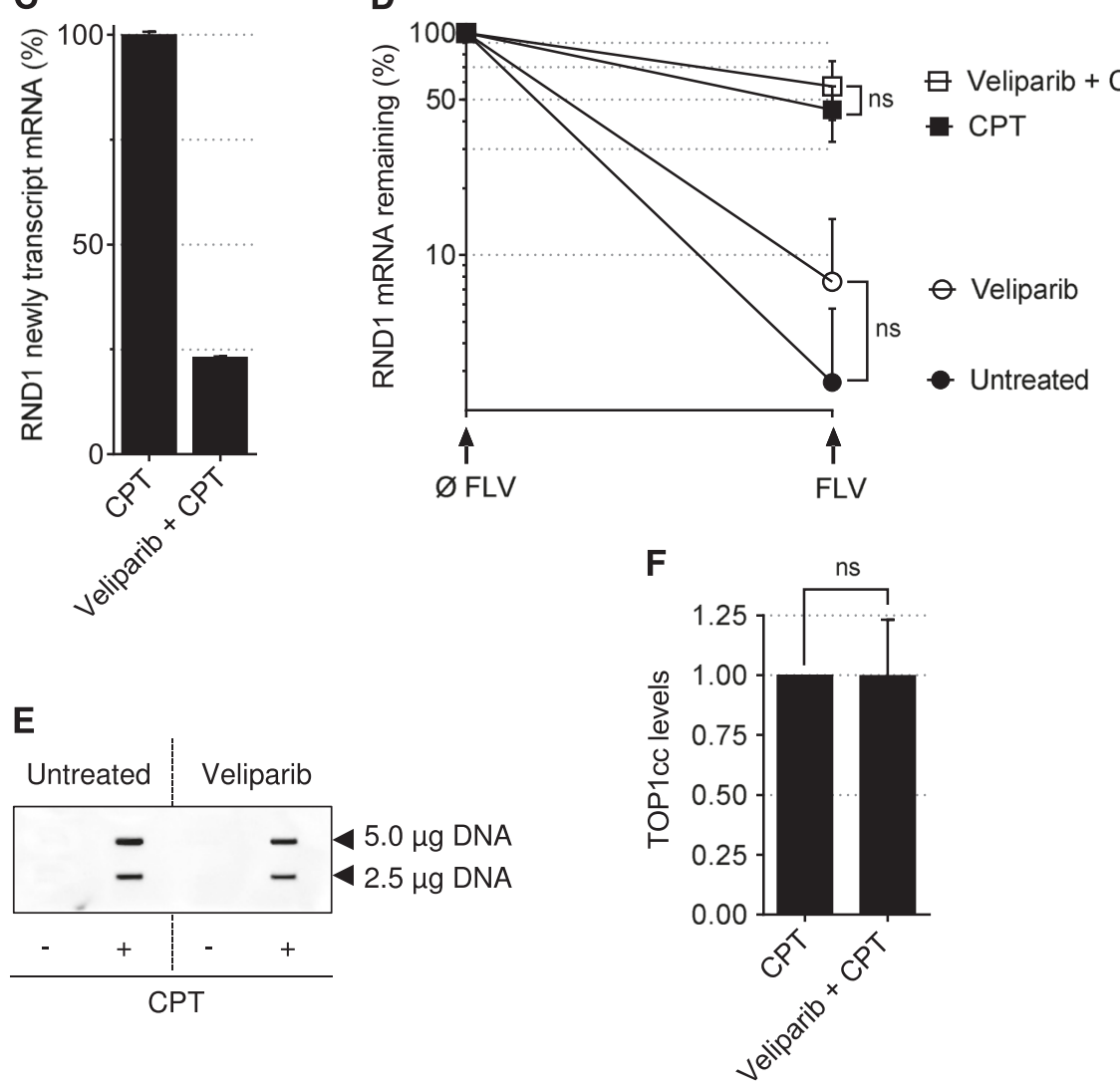

Fig. 4 (See legend on next page.)

activity $^{31}$, we examined whether PARP-1 could promote the increase in RND1 transcript levels in CPT-treated cells.
As reported ${ }^{31}$, CPT increased protein PARylation (Fig. 4a). Protein PARylation was reversible and returned to its baseline level after CPT removal (Fig. 4a), a similar 
(see figure on previous page)

Fig. 4 The PARP-1 inhibitor veliparib prevents CPT-induced RND1 transcription. a U2OS cells were treated with $25 \mu \mathrm{M}$ CPT for $2 \mathrm{~h}$ and washed and cultured in CPT-free medium (CPT + Washes) for $2 \mathrm{~h}$ to allow reversion of TOP1cc. When indicated, cells were pretreated with $5 \mu \mathrm{M}$ veliparib for $1 \mathrm{~h}$. The expression of PAR and PARP-1 were analyzed by Western blotting. The top panel shows quantification of PAR normalized to PARP-1 (means $\pm \mathrm{SD}, n=2$ ). $\mathbf{b}$ RT-qPCR analysis of RND1 mRNA in U2OS cells treated with $5 \mu \mathrm{M}$ veliparib for $1 \mathrm{~h}$ before the addition of CPT for $2 \mathrm{~h}$ (means \pm SD, $n \geq$ 3). ${ }^{* *} P<0.01,{ }^{* * *} P<0.0001$ by unpaired $t$ test. $\mathbf{c}$ U2OS cells were treated with $5 \mu \mathrm{M}$ of veliparib for $1 \mathrm{~h}$ before the addition of $25 \mu \mathrm{M}$ CPT for $2 \mathrm{~h}$. At the end of each time point, $0.2 \mathrm{mM}$ EU was added to the culture medium for $2 \mathrm{~h}$, after which EU-labeled nascent RNAs were captured. Nascent RND1 RNAs were then analyzed by RT-qPCR. Data were normalized to the level of CPT-treated cells, which was taken at 100\%. A representative experiment out of two is shown (means \pm SD of triplicate samples). $\mathbf{d}$ U2OS cells were treated with veliparib $(5 \mu \mathrm{M}, 1 \mathrm{~h})$ followed by the addition of CPT ( $25 \mu \mathrm{M}, 1$ h). After which, the transcription inhibitor FLV $(1 \mu \mathrm{M})$ was added for $4 \mathrm{~h}$. RND1 mRNA expression was analyzed by RT-qPCR and normalized to the level at the time of FLV addition, which was set to $100 \%$ (means $\pm S D, n=3$ ). Ns, not significant by two-way ANOVA. e, f U2OS cells were treated with $5 \mu \mathrm{M}$ veliparib for $1 \mathrm{~h}$ before the addition of $25 \mu \mathrm{M}$ CPT for $2 \mathrm{~h}$, and TOP1cc were detected by probing two concentrations of genomic DNA (5 and $2.5 \mu \mathrm{g}$ ) with an anti-TOP1cc antibody. e Representative experiment. $\mathbf{f}$ Quantification of TOP1cc in veliparib + CPT-treated cells normalized to values from CPT-treated cells (means \pm SEM, $n=3$ ). Ns, not significant by unpaired $t$ test.
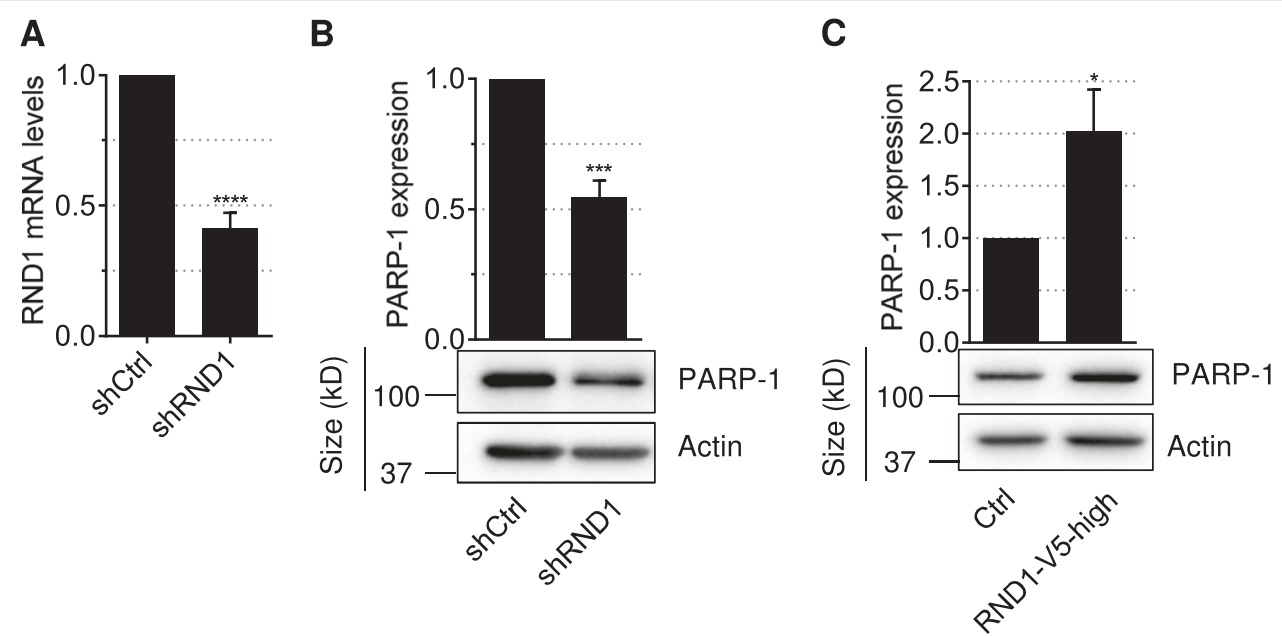

Fig. 5 RND1 expression regulates PARP-1 expression. $\mathbf{a}$, $\mathbf{b} \cup 2 \mathrm{OS}$ cells were stably expressing shRNAs against RND1 (shRND1) or against a control sequence (shCtrl). a RT-qPCR analysis of RND1 mRNA (means \pm SD, $n=4$ ). ${ }^{* * * P}<0.0001$ by unpaired $t$ test. $\mathbf{b}$ Western blotting analysis of PARP-1. The top panel shows quantification of PARP-1 normalized to actin or tubulin (means $\pm \mathrm{SD}, n=5$ ). ${ }^{* * *} P<0.001$ by unpaired $t$ test. $\mathbf{c}$ Western blotting analysis of PARP-1 in U2OS stably expressing high levels of V5-tagged RND1 (RND1-V5-high, see panel Fig. 1g, h) or EGFP (ctrl). The top panel shows quantification of PARP-1 normalized to actin (means $\pm \mathrm{SD}, n=3$ ). ${ }^{*} P<0.05$ by unpaired $t$ test.

effect to that of RND1 mRNA levels (Fig. 2c) and RND1 gene transcription (Fig. 3a). Then, we assessed whether inhibiting PARP-1 activity would prevent the induction of RND1 mRNA. The PARP-1 inhibitor veliparib partially prevented the induction of RND1 mRNA in response to CPT (Fig. 4b) under conditions where it prevented protein PARylation (Fig. 4a). Then, we asked whether PARP inhibition would decrease RND1 transcription and/or RND1 transcript stability. In CPT-treated cells, veliparib strongly inhibited RND1 transcription (Fig. 4c), while it did not decrease the half-life of RND1 transcripts (Fig. 4d). As previously reported ${ }^{38}$, veliparib did not affect TOP1cc levels in response to CPT (Fig. 4e, f), which further suggests that PARP-1 is downstream from TOP1cc to increase RND1 transcription. Altogether, these results suggest that, in CPT-treated cells, TOP1cc stabilization increases PARP-1 activity, which in turn increases the transcription of $R N D 1$ gene, leading to an increase of RND1 transcripts.

\section{RND1 increases PARP-1 expression in a positive feedback loop}

Next, we considered whether there is a cross-talk between PARP-1 and RND1 or whether the talk is limited to one direction in which PARP-1 induces RND1. To test this, we asked whether modulating RND1 expression would modulate PARP-1 expression. Downregulation of RND1 mRNA levels by shRNA in U2OS cells (Fig. 5a), decreased PARP-1 expression (Fig. 5b). Conversely, U2OS cells overexpressing RND1 (characterized in Fig. 1g, h), also overexpressed PARP-1 (Fig. 5c). These results suggest that PARP-1 activity increases RND1, which in turn increases PARP-1 expression in a positive feedback loop. 


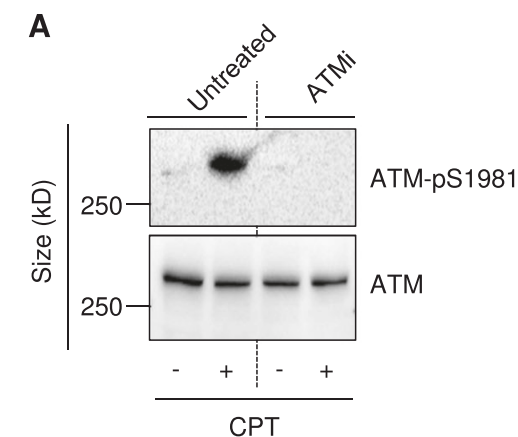

B

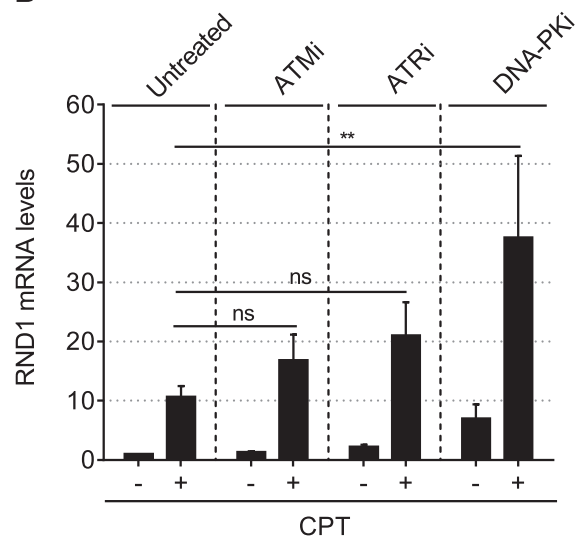

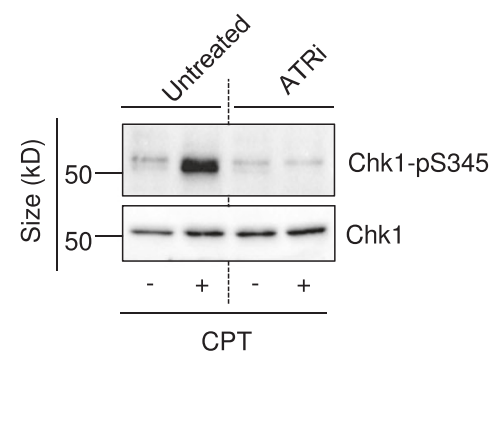

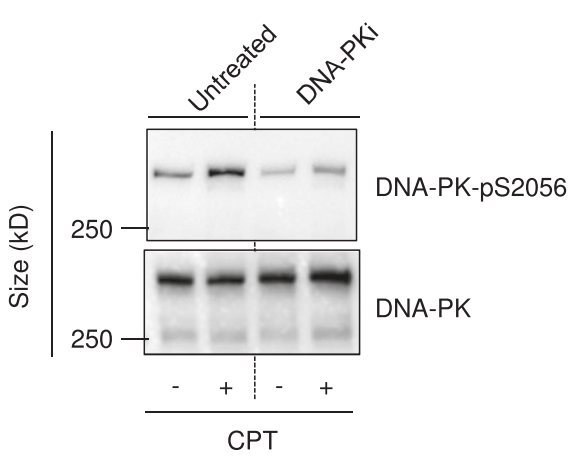

C

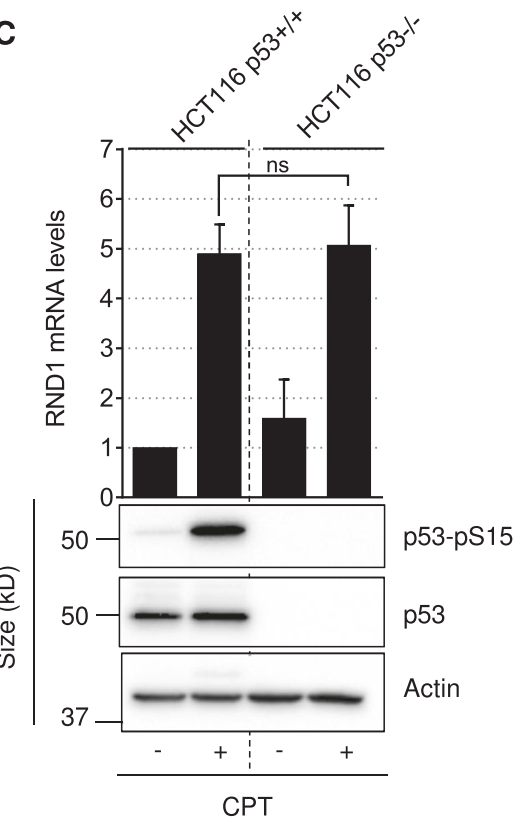

Fig. 6 DNA-PK inhibition increases the induction of $R$ ND1 transcripts by CPT. $\mathbf{a}$, $\mathbf{b} \cup 2 \mathrm{OS}$ cells were treated with ATMi $(10 \mu \mathrm{M})$, ATRi (10 $\mu \mathrm{M})$ or DNA-PKi $(10 \mu \mathrm{M})$ for $1 \mathrm{~h}$ before the addition of $25 \mu \mathrm{M}$ CPT for $2 \mathrm{~h}$. a Western blotting analysis of the indicated proteins. $\mathbf{b}$ RT-qPCR analysis of RND1 mRNA (means \pm SEM, $n \geq 3$ ), ${ }^{* *} P<0.01$, by one-way ANOVA. $\mathbf{c ~ H C T 1 1 6 ~ p 5 3 + / + ~ a n d ~ H C T 1 1 6 ~ p 5 3 - / - ~ c e l l s ~ w e r e ~ t r e a t e d ~ w i t h ~} 25 \mu \mathrm{M} \mathrm{CPT} \mathrm{for} 2 \mathrm{~h}$. Top panel: RND1 mRNA was analyzed by RT-qPCR (means \pm SEM, $n=3$ ). Ns, not significant by one-way ANOVA. Bottom panel: Western blotting analysis of p53-p515 and p53. Actin: loading control. Ns, not significant.

\section{DNA-PK-dependent DSB signaling prevents the induction of RND1 transcripts by CPT}

Because CPT-induced TOP1cc can lead to the production of DSBs ${ }^{19-2122-24}$, we examined the role of DSB signaling in the induction of RND1. ATM, ATR and DNA-PK are serine/threonine kinases that are readily activated by DSBs, and phosphorylate various DNA damage response proteins such as $\mathrm{p} 53^{51}$. Consistent with that, CPT activated these three kinases in U2OS cells as demonstrated by autophosphorylation of ATM at S1981 (ATM-pS1981), phosphorylation of the ATR substrate Chk1 at S345 (Chk1-pS345), and autophosphorylation of DNA-PK at S2056 (Fig. 6a). To determine their potential role in RND1 induction, we assessed whether RND1 induction is modified by specific chemical inhibitors of these kinases in CPT-treated U2OS cells: the ATM inhibitor (ATMi) KU55933, the ATR inhibitor (ATRi) VE-821 and the DNA-PK inhibitor (DNA-PKi) NU7441 (Fig. 6a). Figure 6b shows that DNA-PKi, and in a lesser extend ATMi and ATRi, increased the induction of RND1 mRNA in response to CPT. Because p53 is phosphorylated and activated by these kinases ${ }^{51}$, we examined RND1 induction in $p 53+/+$ and $p 53-/-$ HCT116 cells exposed to CPT. As expected, CPT induced p53 phosphorylation at $\mathrm{S} 15$ and increased p53 protein level in $p 53+/+$ HCT116 cells (Fig. 6c, bottom panels). We found that $p 53$ $+/+$ and $p 53-/-$ HCT116 cells both displayed similar induction of RND1 mRNA in response to CPT (Fig. 6c, top panel). Collectively, our experiments suggest that the DNA-PK-dependent DSB signaling prevents the induction of RND1 transcripts by CPT. However, this DSB signaling pathway is not mediated by $\mathrm{p} 53$. 


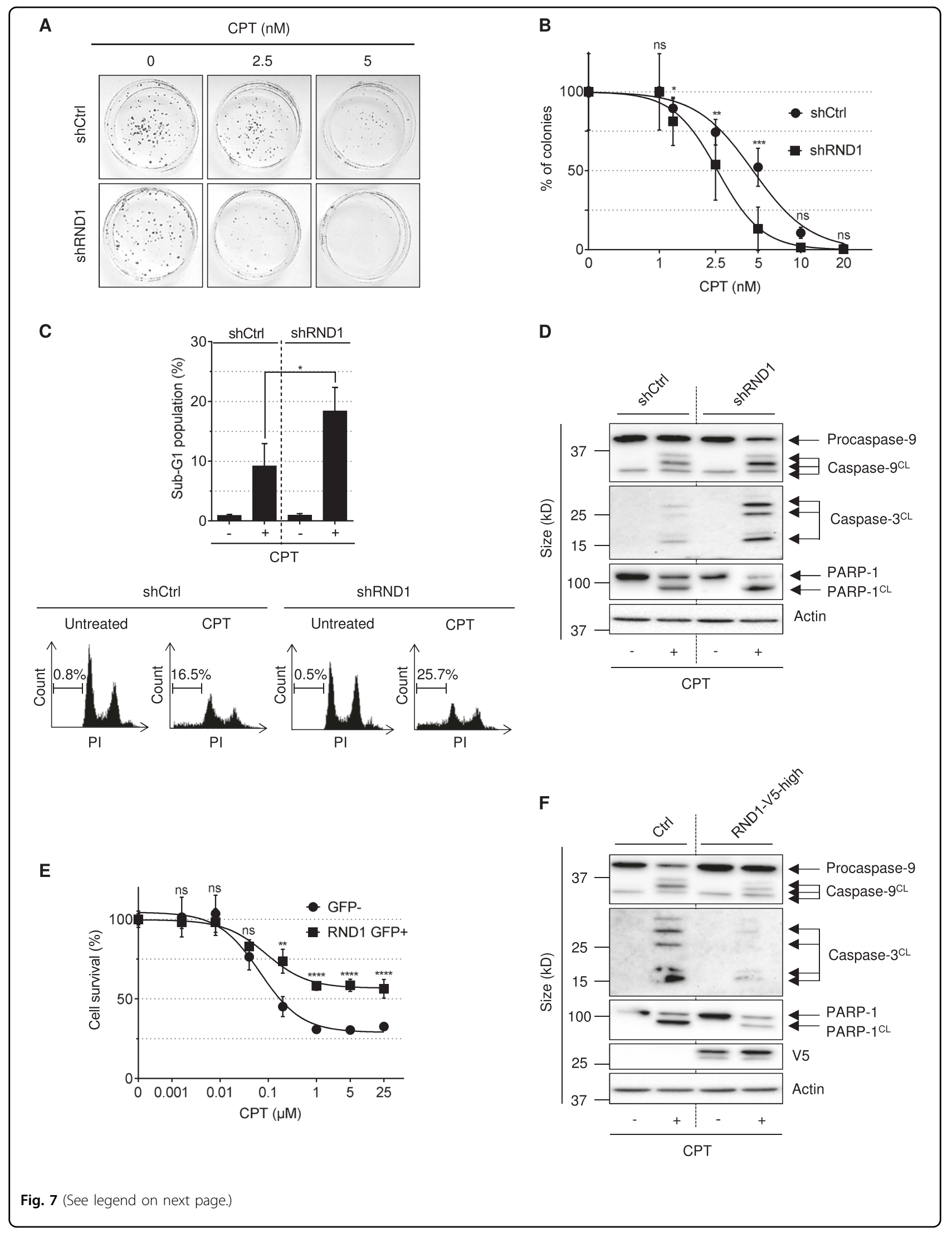


(see figure on previous page)

Fig. 7 RND1 protects cells against CPT-induced apoptosis. a, b Colony formation assay in U2OS cells stably expressing shRNAs against RND1 (shRND1) or against a control sequence (shCtrl), and treated with increasing concentrations of CPT (from 1.25 to $20 \mathrm{nM}$ ). Percentages of colonies were assessed after 10 days by counting the number of colonies and normalized to that of untreated cells, which was set at 100\% (means \pm SD, $n=4$ ), ${ }^{*} P<0.05,{ }^{* *} P<0.01,{ }^{* * *} P<0.001$, by two-way ANOVA. c U2OS shRND1 or shCtrl cells were treated with $25 \mu \mathrm{M}$ CPT for $24 \mathrm{~h}$. Percentage of sub-G1 cell population was analyzed by flow cytometry. The top panel shows quantification of sub-G1 cell population (means \pm SEM, $n=3$ ). ${ }^{*} P<0.05$ by unpaired $t$ test. Bottom: one representative experiment is shown. $\mathbf{d}$ Western blotting analysis of the indicated proteins in U2OS shRND1 or shCtrl cells treated with $25 \mu \mathrm{M}$ CPT for $24 \mathrm{~h}$. Caspase-9 ${ }^{\mathrm{CL}}$ : cleaved caspase-9, Caspase-3 ${ }^{\mathrm{CL}}$ : cleaved caspase-3, PARP-1 ${ }^{\mathrm{CL}}$ : cleaved PARP-1. Data shown are representatives from three experiments. (e) U2OS cells were transfected with pEGFP-RND1 plasmid. Forty-eight hours after transfection, GFP- and GFP + U2OS cells were separated by cell sorting. GFP - and RND1 GFP+ U2OS cells were treated with increasing concentrations of CPT (from $0.0016 \mu \mathrm{M}$ to $25 \mu \mathrm{M}$ ). Seventy-two hours after treatment, cell survival was analyzed by a WST-1 assay. A representative experiment out of three is shown (means \pm SD for triplicate samples). Ns not significant, ${ }^{* *} P<0.01,{ }^{* * *} P<0.0001$, by two-way ANOVA. f Similar experiments as in panel (d) in U2OS Ctrl and RND1-V5high cells. Ns not significant

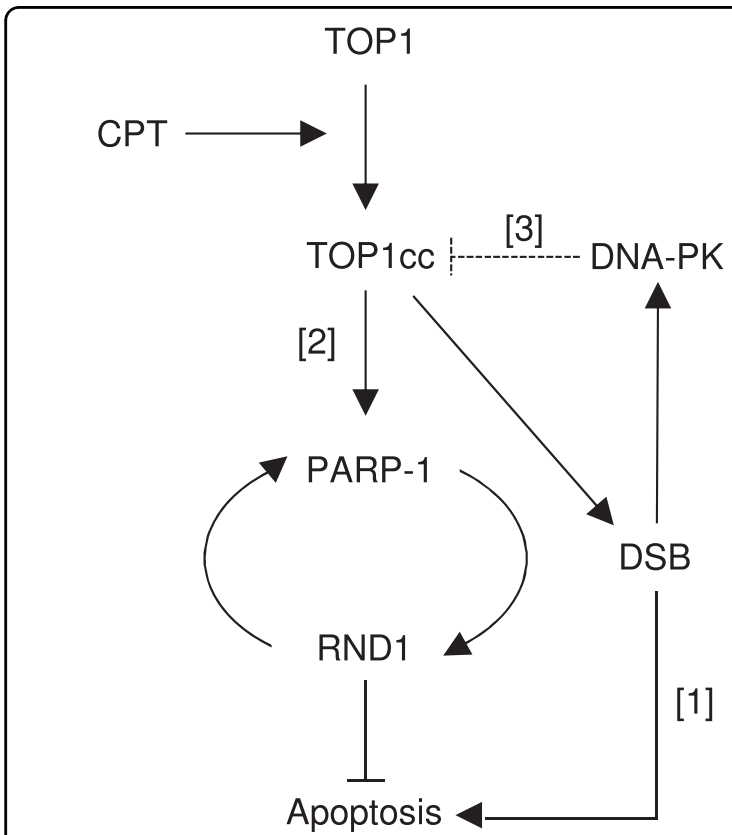

Fig. 8 Proposed model for the induction of RND1 in response to CPT. CPT stabilizes TOP1cc, which in turn induce DSBs and apoptosis $^{25}$ [1]. TOP1cc also activates a PARP-1-RND1 pathway that counteracts the induction of apoptosis [2]. DNA-PK-dependent DSB signaling prevents RND1 induction, possibly by promoting TOP1cc removal $^{22,52}[3]$

\section{RND1 reduces the sensitivity of cells exposed to CPT}

To assess the potential role of RND1 in the cellular response to TOP1cc stabilization, we first compared the sensitivity of shCtrl and shRND1 U2OS cells (characterized in Fig. 5a) to CPT treatment. Cells were treated with increasing concentrations of $\mathrm{CPT}$, and $\mathrm{CPT}$ sensitivity was assessed by clonogenic assays. Figure $7 \mathrm{a}$, b shows that shRND1 cells formed significantly less clones in response to CPT than shCtrl cells. The increased sensitivity of shRND1 cells to CPT was further associated with an increase of apoptotic marks such as sub-G1 population (Fig. 7c), and the cleavage of caspase-9, caspase-3 and PARP-1 (Fig. 7d). Conversely, overexpression of RND1 in
U2OS cells (Supplementary Figure 3) decreased cell sensitivity to CPT as measured by WST-1 survival assays (Fig. 7e) and decreased apoptotic marks (Fig. 7f). Together, these results demonstrate that RND1 protects cells against $\mathrm{CPT}$, likely by preventing apoptosis.

\section{Discussion}

Here we identified RND1 as an early inducible RHO GTPase gene in response to CPT. This is the first time that an atypical RHO is reported to respond early to DNA damaging agents. Our data support a model depicted in Fig. 8 in which CPT-induced TOP1cc stabilization increases PARP-1 activity that triggers RND1 transcription, which elevates the levels of RND1 transcripts (and likely also the protein). In turn, the increase of RND1 protein levels promotes an increase of PARP-1 protein levels, suggesting a positive feedback loop between PARP1 and RND1 in response to CPT. The increase of RND1 induced by the TOP1cc-PARP-1 pathway protects cells against CPT, likely by inhibiting apoptosis. PARP-1independent pathways probably also contribute to the increase of RND1 transcript levels, as the inhibition of PARP-1 activity with veliparib does not completely suppress CPT-induced RND1 transcripts. Such pathways might involve an increased stability of RND1 transcripts as our analysis shows that CPT extends the half-life of RND1 mRNA in a PARP-1-independent manner. CPTinduced TOP1cc also induce DSBs, which activate DNAPK that reduces the induction of RND1. DNA-PK could reduce the induction of RND1 by promoting TOP1 proteolysis as previously reported ${ }^{22,52}$. Albeit in a lesser extent, ATM, which can activate DNA-PK ${ }^{22}$, also reduces the induction of RND1. Consistent with that, ATM has also been reported to promote TOP1 proteolysis ${ }^{53,54}$.

Our study uncovers the close relationship between TOP1cc and the transcription of RND1. Indeed, CPT, which induces $R N D 1$ transcription, has for sole cellular target the TOP1cc ${ }^{14}$, and reversion of TOP1cc following termination of the CPT treatment readily restores the 
baseline levels of RND1 transcription and RND1 transcripts. In addition, $\mathrm{H}_{2} \mathrm{O}_{2}$ and UV light, which induce DNA lesions that interfere with TOP1 nicking-closing activity and give rise to elevated levels of TOP1 $\mathrm{cc}^{15-18,40}$, also increase RND1 transcript levels. Besides TOP1 inhibitors and DNA alterations (see Table 1 in ref. ${ }^{13}$ ), several other processes lead to persistent TOP1cc, including ribonucleotide incorporation into $\mathrm{DNA}^{55-57}$, genetic defects such as ATM defect ${ }^{53,54}$, and transcriptional activation ${ }^{58}$. Hence, the increased transcription of RND1 due to TOP1cc stabilization might be a frequent event occurring under both physiological and stress conditions.

An early response to CPT is the global inhibition of transcription $^{22,45}$. However, genes are differentially affected by CPT and a fraction of them, primarily the short and low-expressed genes, are upregulated ${ }^{27-29}$. In accordance with this, $R N D 1$ is a short gene $(8.7 \mathrm{Kbp})$, with a lowexpression in most healthy tissues apart from brain and liver $^{2}$ and in addition, RND1 expression is significantly downregulated in several aggressive tumors compared to normal tissues ${ }^{39,59,60}$. The mechanisms by which CPT enhances transcription of some genes are largely unknown. Here we reported that CPT induces PARP-1 activity, which in turn stimulates $R N D 1$ transcription. This effect is likely related to TOP1cc. Similar to CPT, $\mathrm{H}_{2} \mathrm{O}_{2}$ and UV light induce persistent TOP1cc ${ }^{18,40}$, increase RND1 transcript levels (this study), and also increase PARP-1 activity ${ }^{61,62}$. Whether TOP1cc-induced PARP-1 activity is a common mechanism for CPT to promote gene transcription or whether it is restricted to RND1 gene remains to be investigated.

It is now well documented that PARP-1 regulates transcription $^{63}$ asides from its well-recognized role in DNA repair ${ }^{64}$. PARP-1 is enriched to the promoters of actively transcribed genes ${ }^{65}$ and, stimulates transcription initiation by maintaining an 'open' chromatin environment through PARylation of core histones and exclusion of histone $\mathrm{H} 1$ from the $\mathrm{DNA}^{48,65}$, or inhibition of histone H3K4me demethylation by KDM5B ${ }^{47}$. PARP-1 could also promote transcription by stimulating transcription elongation. PARP-1 PARylates subunits of the negative elongation factors (NELF), NELF-A and NELF-E, which triggers the release of RNA polymerase II from its paused site for productive elongation ${ }^{49}$. Our results showing that increased RND1 transcription by CPT does not relies on increased activity of its promoter suggest that PARP-1 might primarily function in stimulating transcription elongation of RND1 gene. PARP-1-independent pathways probably also contribute to the increase of RND1 transcription, as PARP-1 inhibition does not completely suppress CPT-induced $R N D 1$ transcription. A previous study shows that the CPT derivatives topotecan can stimulate $U B E 3 A$ transcription by downregulating the expression of its antisense transcript ${ }^{66}$. The non-coding
RNA AGAP2-AS1 has been reported to inhibit RND1 transcription $^{67,68}$, which raises the possibility that CPT could inhibit AGAP2-AS1 transcription, which in turn could increase RND1 transcription.

Lastly, our analysis shows that RND1 protects cells against CPT-induced apoptosis and hence favors cell resistance. These findings extend the role of RND1 beyond its original function in the disassembly of actin filament structures and loss of cell adhesion ${ }^{2}$ as well as in embryonic development, where it promotes the formation and maturation of neuronal protrusions ${ }^{69,70}$ and controls gastrulation movements ${ }^{71}$. In addition, RND1 behaves as a tumor suppressor gene. RND1 expression levels decrease in several aggressive tumors ${ }^{39,59,60}$, and RND1 loss in immortalized mammary cells can initiate breast tumorigenesis and promotes metastasis ${ }^{39}$. Even in tumor cell lines expressing low levels of RND1 such as MCF-7 cells $^{39}$, U87 cells ${ }^{59}$ and U2OS cells, RND1 could be transiently induced by TOP1cc to resist to CPT derivatives. This potential selective advantage of tumor cells suggests that inhibiting RND1-dependent signaling could sensitize them to $\mathrm{CPT}$ derivatives.

\section{Acknowledgements \\ We thank E. Nicolas for WI38 hTERT cells, Dr Tan for pGL3-basal promoterenhancer1RND1-lucF, Dr Minami for pGL3-basalpromoterRND1-lucF and pGL3-promoterenhancer2RND1-lucF, Pr Moyal and Dr Toulas for the lentiviral particles. We thank Manon Farce for her kind technical assistance. This work was supported by the Ligue Nationale Contre le Cancer (LNCC) Comité Départemental 31; the Institut National de la Santé et de la Recherche Médicale (INSERM); the Ministère de l'Enseignement Supérieur et de la Recherche [doctoral fellowship to A.C.]; the Fondation pour la Recherche Médicale (FRM) [FDT20140931166; doctoral fellowship to A.C.]; the Région Midi-Pyrénées and INSERM [R14075BB doctoral fellowship to L.M.].}

Conflict of interest

The authors declare that they have no conflict of interest

\section{Publisher's note}

Springer Nature remains neutral with regard to jurisdictional claims in published maps and institutional affiliations.

Supplementary Information accompanies this paper at (https://doi.org/ 10.1038/s41419-018-0981-3).

Received: 20 March 2018 Revised: 2 August 2018 Accepted: 20 August 2018 Published online: 12 September 2018

\footnotetext{
References

1. Haga, R. B. \& Ridley, A. J. Rho GTPases: regulation and roles in cancer cell biology. Small GTPases 7, 207-221 (2016)

2. Nobes, C. D. et al. A new member of the Rho family, Rnd1, promotes disassembly of actin filament structures and loss of cell adhesion. J. Cell Biol. 141, 187-197 (1998).

3. Riou, P., Villalonga, P. \& Ridley, A. J. Rnd proteins: multifunctional regulators of the cytoskeleton and cell cycle progression. Bioessays 32, 986-992 (2010).

4. Shutes, A., Berzat, A. C., Cox, A. D. \& Der, C. J. Atypical mechanism of regulation of the Wrch-1 Rho family small GTPase. Curr. Biol. 14, 2052-2056 (2004).

5. Lawson, C. D. \& Ridley, A. J. Rho GTPase signaling complexes in cell migration and invasion. J. Cell Biol. 217, 447-457 (2018).
} 
6. Deshmukh, J., Pofahl, R. \& Haase, I. Epidermal Rac1 regulates the DNA damage response and protects from UV-light-induced keratinocyte apoptosis and skin carcinogenesis. Cell Death Dis. 8, e2664 (2017).

7. Yan, Y., Greer, P. M., Cao, P. T., Kolb, R. H. \& Cowan, K. H. RAC1 GTPase plays an important role in gamma-irradiation induced G2/M checkpoint activation. Breast Cancer Res. 14, R60 (2012).

8. Espinha, G., Osaki, J. H., Magalhaes, Y. T. \& Forti, F. L. Rac1 GTPase-deficient HeLa cells present reduced DNA repair, proliferation, and survival under UV or gamma irradiation. Mol. Cell Biochem. 404, 281-297 (2015).

9. Mamouni, K et al. RhoB promotes gammaH2AX dephosphorylation and DNA double-strand break repair. Mol. Cell Biol. 34, 3144-3155 (2014).

10. Canguilhem, B. et al. RhoB protects human keratinocytes from UVB-induced apoptosis through epidermal growth factor receptor signaling. J. Biol. Chem. 280, 43257-43263 (2005).

11. Fritz, G., Kaina, B. \& Aktories, K. The ras-related small GTP-binding protein RhoB is immediate-early inducible by DNA damaging treatments. J. Biol. Chem. 270, 25172-25177 (1995).

12. Meyer, N. et al. RhoB Promotes Cancer Initiation by Protecting Keratinocytes from UVB-Induced Apoptosis but Limits Tumor Aggressiveness. J. Invest. Dermatol. 134, 203-212 (2014).

13. Pommier, Y., Sun, Y., Huang, S. N. \& Nitiss, J. L. Roles of eukaryotic topoisomerases in transcription, replication and genomic stability. Nat. Rev. Mol. Cell Biol. 17, 703-721 (2016).

14. Pommier, Y. Topoisomerase I inhibitors: camptothecins and beyond. Nat. Rev. Cancer 6, 789-802 (2006).

15. Pourquier, P. et al. Induction of reversible complexes between eukaryotic DNA topoisomerase I and DNA-containing oxidative base damages. J. Biol. Chem. 274, 8516-8523 (1999).

16. Lesher, D. T., Pommier, Y., Stewart, L. \& Redinbo, M. R. 8-Oxoguanine rearranges the active site of human topoisomerase I. Proc. Natl Acad. Sci. USA 99 12102-12107 (2002)

17. Lanza, A., Tornaletti, S., Rodolfo, C., Scanavini, M. C. \& Pedrini, A. M. Human DNA topoisomerase I-mediated cleavages stimulated by ultraviolet light-induced DNA damage. J. Biol. Chem. 271, 6978-6986 (1996).

18. Subramanian, D., Rosenstein, B. S. \& Muller, M. T. Ultraviolet-induced DNA damage stimulates topoisomerase I-DNA complex formation in vivo: possible relationship with DNA repair. Cancer Res. 58, 976-984 (1998).

19. Furuta, T. et al. Phosphorylation of histone H2AX and activation of Mre11, Rad50, and Nbs1 in response to replication-dependent DNA double-strand breaks induced by mammalian DNA topoisomerase I cleavage complexes. J. Biol. Chem. 278, 20303-20312 (2003).

20. Regairaz, M. et al. Mus81-mediated DNA cleavage resolves replication forks stalled by topoisomerase I-DNA complexes. J. Cell Biol. 195, 739-749 (2011).

21. Strumberg, D. et al. Conversion of topoisomerase I cleavage complexes on the leading strand of ribosomal DNA into 5'-phosphorylated DNA double-strand breaks by replication runoff. Mol. Cell Biol. 20, 3977-3987 (2000).

22. Cristini, A. et al. DNA-PK triggers histone ubiquitination and signaling in response to DNA double-strand breaks produced during the repair of transcription-blocking topoisomerase | lesions. Nucleic Acids Res. 44, 1161-1178 (2016)

23. Sordet, O., Nakamura, A. J., Redon, C. E. \& Pommier, Y. DNA double-strand breaks and ATM activation by transcription-blocking DNA lesions. Cell Cycle $\mathbf{9}$, 274-278 (2010).

24. Sordet, O. et al. Ataxia telangiectasia mutated activation by transcription- and topoisomerase l-induced DNA double-strand breaks. EMBO Rep. 10, 887-893 (2009).

25. Sordet, O., Khan, Q. A., Kohn, K. W. \& Pommier, Y. Apoptosis induced by topoisomerase inhibitors. Curr. Med. Chem. AntiCancer Agents 3, 271-290 (2003).

26. Capranico, G. et al. The effects of camptothecin on RNA polymerase II transcription: roles of DNA topoisomerase I. Biochimie 89, 482-489 (2007)

27. Solier, S. et al. Transcription poisoning by Topoisomerase I is controlled by gene length, splice sites, and miR-142-3p. Cancer Res. 73, 4830-4839 (2013).

28. Veloso, A. et al. Genome-wide transcriptional effects of the anti-cancer agent camptothecin. PLOS ONE 8, e78190 (2013).

29. King, I. F. et al. Topoisomerases facilitate transcription of long genes linked to autism. Nature 501, 58-62 (2013).

30. Jeanblanc, M. et al. Parallel pathways in RAF-induced senescence and conditions for its reversion. Oncogene 31, 3072-3085 (2012).

31. Das, B. B. et al. PARP1-TDP1 coupling for the repair of topoisomerase l-induced DNA damage. Nucleic Acids Res. 42, 4435-4449 (2014).
32. Monferran, S. et al. Alphavbeta3 and alphavbeta5 integrins control glioma cell response to ionising radiation through ILK and RhoB. Int. J. Cancer 123, 357-364 (2008).

33. Davis, M. et al. Toxicogenomics profiling of bone marrow from rats treated with topotecan in combination with oxaliplatin: a mechanistic strategy to inform combination toxic ty. Front. Genet. 6, (2015).

34. Bansal, M. et al. A community computational challenge to predict the activity of pairs of compounds. Nat. Biotechnol. 32, 1213-U1269 (2014).

35. lorio, F. et al. Discovery of drug mode of action and drug repositioning from transcriptional responses. Proc. . Natl Acad. Sci. U. S. A. 107, 14621-14626 (2010).

36. Lamb, J. et al. The connectivity map: Using gene-expression signatures to connect small molecules, genes, and disease. Science 313, 1929-1935 (2006).

37. Patel, A. G. et al. Immunodetection of human topoisomerase I-DNA covalent complexes. Nucleic Acids Res. 44, 2816-2826 (2016).

38. Zhang, Y. W. et al. Poly(ADP-ribose) polymerase and XPF-ERCC1 participate in distinct pathways for the repair of topoisomerase l-induced DNA damage in mammalian cells. Nucleic Acids Res. 39, 3607-3620 (2011).

39. Okada, T. et al. The Rho GTPase Rnd1 suppresses mammary tumorigenesis and EMT by restraining Ras-MAPK signalling. Nat. Cell Biol. 17, 81-94 (2015).

40. Daroui, P., Desai, S. D., Li, T. K., Liu, A. A. \& Liu, L. F. Hydrogen peroxide induces topoisomerase I-mediated DNA damage and cell death. J. Biol. Chem. 279, 14587-14594 (2004).

41. Covey, J. M., Jaxel, C., Kohn, K. W. \& Pommier, Y. Protein-linked DNA strand breaks induced in mammalian cells by camptothecin, an inhibitor of topoisomerase I. Cancer Res. 49, 5016-5022 (1989).

42. Shen, L. et al. Overexpression of Oct4 suppresses the metastatic potential of breast cancer cells via Rnd1 downregulation. Biochim. Biophys. Acta 1842 2087-2095 (2014).

43. Suehiro, J. et al. Genome-wide approaches reveal functional vascular endothelial growth factor (VEGF)-inducible nuclear factor of activated T cells (NFAT) C1 binding to angiogenesis-related genes in the endothelium. J. Biol. Chem. 289, 29044-29059 (2014).

44. Sordet, O. et al. Hyperphosphorylation of RNA polymerase II in response to topoisomerase I cleavage complexes and its association with transcriptionand BRCA1-dependent degradation of topoisomerase I. J. Mol. Biol. 381, 540-549 (2008).

45. Desai, S. D. et al. Transcription-dependent degradation of topoisomerase IDNA covalent complexes. Mol. Cell Biol. 23, 2341-2350 (2003).

46. Kim, M. Y., Mauro, S., Gevry, N., Lis, J. T. \& Kraus, W. L. NAD+-dependent modulation of chromatin structure and transcription by nucleosome binding properties of PARP-1. Cell 119, 803-814 (2004).

47. Krishnakumar, R. \& Kraus, W. L. PARP-1 regulates chromatin structure and transcription through a KDM5B-dependent pathway. Mol. Cell 39, 736-749 (2010).

48. Martinez-Zamudio, R. \& Ha, H. C. Histone ADP-ribosylation facilitates gene transcription by directly remodeling nucleosomes. Mol. Cell Biol. 32, 2490-2502 (2012).

49. Gibson, B. A. et al. Chemical genetic discovery of PARP targets reveals a role for PARP-1 in transcription elongation. Science 353, 45-50 (2016).

50. Ke, Y. et al. PARP1 promotes gene expression at the post-transcriptiona level by modulating the RNA-binding protein HuR. Nat. Commun. 8, 14632 (2017).

51. Blackford, A. N. \& Jackson, S. P. ATM, ATR, and DNA-PK: the trinity at the heart of the DNA damage response. Mol. Cell 66, 801-817 (2017).

52. Ando, $\mathrm{K}$. et al. Camptothecin resistance is determined by the regulation of topoisomerase I degradation mediated by ubiquitin proteasome pathway. Oncotarget 8, 43733-43751 (2017).

53. Katyal, S. et al. Aberrant topoisomerase-1 DNA lesions are pathogenic in neurodegenerative genome instability syndromes. Nat. Neurosci. 17, 813-821 (2014).

54. Alagoz, M., Chiang, S. C., Sharma, A. \& El-Khamisy, S. F. ATM deficiency results in accumulation of DNA-topoisomerase I covalent intermediates in neural cells. PLoS. One. 8, e58239 (2013).

55. Huang, S. Y., Ghosh, S. \& Pommier, Y. Topoisomerase I alone is sufficient to produce short DNA deletions and can also reverse nicks at ribonucleotide sites. J. Biol. Chem. 290, 14068-14076 (2015).

56. Kim, N. et al. Mutagenic processing of ribonucleotides in DNA by yeast topoisomerase I. Science 332, 1561-1564 (2011).

57. Sparks, J. L. \& Burgers, P. M. Error-free and mutagenic processing of topoisomerase 1-provoked damage at genomic ribonucleotides. EMBO J. 34 1259-1269 (2015) 
58. Puc, J. et al. Ligand-dependent enhancer activation regulated by topoisomerase-l activity. Cell 160, 367-380 (2015).

59. Clarke, K. et al. Inference of low and high-grade glioma gene regulatory networks delineates the role of Rnd3 in establishing multiple hallmarks of cancer. PLoS Genet. 11, e1005325 (2015).

60. Komatsu, $\mathrm{H}$. et al. Attenuated RND1 expression confers malignant phenotype and predicts poor prognosis in hepatocellular carcinoma. Ann. Surg. Oncol. 24, 850-859 (2017).

61. Fujimoto, M. et al. The HSF1-PARP13-PARP1 complex facilitates DNA repair and promotes mammary tumorigenesis. Nat. Commun. 8, 1638 (2017).

62. Jungmichel, S. et al. Proteome-wide identification of poly(ADP-Ribosyl)ation targets in different genotoxic stress responses. Mol. Cell 52, 272-285 (2013).

63. Gupte, R., Liu, Z. \& Kraus, W. L. PARPs and ADP-ribosylation: recent advances linking molecular functions to biological outcomes. Genes Dev. 31, 101-126 (2017).

64. Ray Chaudhuri, A. \& Nussenzweig, A. The multifaceted roles of PARP1 in DNA repair and chromatin remodelling. Nat. Rev. Mol. Cell Biol. 18, 610-621 (2017).
65. Krishnakumar, R. et al. Reciprocal binding of PARP-1 and histone $\mathrm{H} 1$ at promoters specifies transcriptional outcomes. Science 319, 819-821 (2008).

66. Huang, H. S. et al. Topoisomerase inhibitors unsilence the dormant allele of Ube3a in neurons. Nature 481, 185-189 (2012).

67. Li, W. et al. Upregulated long non-coding RNA AGAP2-AS1 represses LATS2 and KLF2 expression through interacting with EZH2 and LSD1 in non-smallcell lung cancer cells. Cell Death Dis. 7, e2225 (2016).

68. Qi, F. et al. Long noncoding AGAP2-AS1 is activated by SP1 and promotes cell proliferation and invasion in gastric cancer. J. Hematol. Oncol. 10, 48 (2017).

69. Ishikawa, Y., Katoh, H. \& Negishi, M. A role of Rnd1 GTPase in dendritic spine formation in hippocampal neurons. J. Neurosci. 23, 11065-11072 (2003).

70. Li, Y. H. et al. Rnd1 regulates axon extension by enhancing the microtubule destabilizing activity of SCG10. J. Biol. Chem. 284, 363-371 (2009).

71. Ogata, S. et al. TGF-beta signaling-mediated morphogenesis: modulation of cell adhesion via cadherin endocytosis. Genes Dev. 21, 1817-1831 (2007). 